\title{
Cisplatin versus carboplatin in combination with paclitaxel as neoadjuvant regimen for triple negative breast cancer
}

This article was published in the following Dove Press journal:

OncoTargets and Therapy

\author{
Liang Huang ${ }^{1,2, *}$ \\ Qi Liu'2,3,* \\ Sheng Chen ${ }^{1,2}$ \\ Zhiming Shao ${ }^{1,2}$
}

'Department of Breast Surgery, Fudan University Shanghai Cancer Center/ Cancer Institute, Shanghai, China; ${ }^{2}$ Department of Oncology, Shanghai Medical College, Fudan University, Shanghai, China; ${ }^{3}$ Department of Radiation Oncology, Fudan University Shanghai Cancer Center/Cancer Institute, Shanghai, China

*These authors contributed equally to this work
Correspondence: Zhiming Shao Department of Breast Surgery, Fudan University Shanghai Cancer Center/ Cancer Institute, 399 Ling-Ling Road, Shanghai, 200032, China Fax +86 2I 64434556

Email zhimingshao@outlook.com
Background: Platinum salts have demonstrated sufficient efficacy and safety for consideration of their use in a neoadjuvant setting for triple negative breast cancer (TNBC).

Patients and methods: We retrospectively analyzed 145 TNBC cases to compare the activity and tolerability of cisplatin and carboplatin. Two groups received weekly paclitaxel and platinum salts.

Results: In total, $87 \%$ of patients in the cisplatin group and $82 \%$ of patients in the carboplatin group experienced a clinical objective response after four cycles (complete response or partial response; $P=0.570$ ). Pathological complete response ( $\mathrm{pCR}$ ) occurred similarly in the cisplatin group and the carboplatin group (44\% versus $42 \%, P=0.789$ ). In survival analysis, there was no difference between the two regimens. The most common grade $3 / 4$ adverse events were neutropenia and leukopenia.

Conclusion: There was no significant difference between the groups in terms of adverse events. Both types of platinum salts and weekly paclitaxel are feasible therapies that achieved high pCR rates and tolerability in TNBC patients.

Keywords: neoadjuvant chemotherapy, triple negative breast cancer, carboplatin, cisplatin, pathological complete response

\section{Introduction}

Triple negative breast cancer (TNBC) is defined as tumors that do not express ER, PR, and HER-2. Treatment options for TNBC have been limited by the lack of traditional targeted therapies, such as hormonal interventions or trastuzumab. Nearly $15 \%$ of breast cancers are defined as TNBC in Western countries, and the vast majority are sporadic. ${ }^{1}$ Most of these tumors are high-grade or poorly differentiated tumors with a high risk of recurrence and mortality.

Neoadjuvant chemotherapy (NCT) has the potential to convert unresectable tumors to resectable ones and can reduce the extent of surgery needed to achieve breast conserving surgery. TNBC shows heterogeneity in response to systemic treatment, which can be divided into six groups according to gene expression profiles. ${ }^{2}$ In the neoadjuvant setting, TNBC can achieve a higher pathological complete response ( $\mathrm{pCR}$ ) rate than the luminal type. ${ }^{3}$ The pCR rates of TNBC can also increase from single-agent to multi-agent neoadjuvant trials. ${ }^{4-6}$ In a pooled analysis, TNBC was associated with a pooled pCR of $34 \% .{ }^{7}$ In a previous meta-analysis, TNBC patients who presented with residual disease after NCT had very poor outcomes. ${ }^{3}$ New therapies should improve the $\mathrm{pCR}$ rate in TNBC patients. A high $\mathrm{pCR}$ rate can benefit survival. 
The previous reports demonstrated that some DNA damaging agents may be more effective in TNBC tumors. ${ }^{8}$ Therefore, there is interest in utilizing DNA-damaging agents, such as platinum drugs (cisplatin and carboplatin) to treat TNBC. Few trials have been designed to compare the efficacy and safety between carboplatin and cisplatin. In a neoadjuvant setting, the addition or lack of platinum versus the same chemotherapy is the most common design. Due to a lack of clinical evidence, the best platinum salt to be added to NCT is still unknown. Here, we retrospectively analyzed 145 patients with locally advanced breast cancer who received a neoadjuvant combination of weekly paclitaxel with cisplatin or carboplatin, to compare the efficacy and safety of the two types of platinum therapy.

\section{Method and treatment Study population}

The cohort in the present study was selected consecutively from patients with locally advanced breast cancer who received NCT followed by surgery at Shanghai Cancer Centre from 2011 to 2015. The major eligibility criteria included: 1) women aged $18-75$ years; 2) ECOG score $0-1$; 3) core needle biopsy-diagnosed invasive breast cancer; 4) hormone receptor-negative (ER and PR negative defined as $<1 \%$ tumor staining by immunohistochemistry [IHC]) and HER-2 negative (IHC: 0 or 1; or IHC: 2 and FISH negative); 5) clinical stage IIA-IIIC with NAC indication and measurable lesions; and 6) normal cardiac, hepatic, and marrow function. Patients were excluded if they had a history of invasive cancer or prior exposure to chemotherapy/radiotherapy. The study was conducted according to the principles expressed in the Declaration of Helsinki and approved by the Institutional Review Board of Fudan University Shanghai Cancer Center/ Cancer Institute. All patients enrolled in this study signed an informed consent voluntarily.

\section{Response and toxicity evaluation}

A pCR was defined as the absence of an invasive tumor in the final surgical breast and axillary lymph node (ALN) sample. Residual ductal carcinoma in situ was included in the pCR category. Standard RECIST guidelines were used to evaluate the clinical and pathological response. No clinical evidence of palpable tumor in the breast and ALNs was defined as a clinical complete response (CR). Reduction in the greatest tumor diameter of $\geq 30 \%$ was graded as a partial response (PR). An increase in greatest tumor diameter of $>20 \%$ or the appearance of new disease was considered as progressive disease (PD). Tumors that did not meet the criteria for objective PR or PD were considered as stable disease (SD).
The Miller-Payne (MP) grading system was employed to evaluate the decrease in cancer cellularity. ${ }^{9}$ Toxicity was evaluated at every period of chemotherapy treatment and based on the NCI-CTCAE v3.0.

\section{Treatment}

In this retrospective study, patients received four cycles of weekly carboplatin (AUC $=2$ calculated using the Calvert formula, d1, d8, d15) and paclitaxel $\left(80 \mathrm{mg} / \mathrm{m}^{2}, \mathrm{~d} 1, \mathrm{~d} 8, \mathrm{~d} 15\right)$ or four cycles of cisplatin $\left(25 \mathrm{mg} / \mathrm{m}^{2}, \mathrm{~d} 1, \mathrm{~d} 8, \mathrm{~d} 15\right)$ treatment and paclitaxel $\left(80 \mathrm{mg} / \mathrm{m}^{2}, \mathrm{~d} 1, \mathrm{~d} 8, \mathrm{~d} 15, \mathrm{~d} 22\right)$. Breast surgery was performed 2-4 weeks after the last chemotherapy dose. The surgery type was at the surgeon's discretion. Adjuvant chemotherapy began within 4 weeks postoperatively. An additional two cycles of the same regimen treatment were administered to patients who achieved pCR; otherwise, four cycles of epirubicin and cyclophosphamide were administered.

\section{Statistical analysis}

Descriptive statistics were calculated to summarize the patient characteristics, tumor size, and biomarker levels according to the core needle biopsies. Toxicity rates in the two cohorts were presented using frequency tabulations with the corresponding percentages. Disease free survival (DFS) was calculated from the date of first diagnosis to the date of disease relapse or metastasis. Overall survival (OS) was calculated from the date of first diagnosis to the date of death or last follow-up. Patients without relapse events or death were censored at the last follow-up. Survival curves were estimated using the Kaplan-Meier method, and the log-rank test was used to determine significance. The Chi-squared test was used to evaluate the relationship between patient characteristics and clinical response. A multivariate logistic regression model for predicting the response to NCT was used when combining a series of variables. All $P$-values reported are two-sided and were calculated at a significance level of 0.05. All statistical procedures were performed using SPSS (version 13.0) and Stata (version 11.0).

\section{Results}

\section{Patient characteristics}

Overall, 145 consecutive patients with breast cancer were enrolled from January 2011 to December 2015 to receive NCT at Fudan University Shanghai Cancer Center. Of these patients, 52 cases were assigned to receive weekly cisplatin and paclitaxel, and 93 cases were assigned to receive weekly carboplatin and paclitaxel. Table 1 summarizes the main clinical and pathological characteristics. These factors were well balanced in the two groups, including age, menopausal status, clinical stage, Ki67 index, and body mass index 
Table I Patient characteristics and clinical evaluation at baseline

\begin{tabular}{|c|c|c|c|}
\hline Characteristics & $\begin{array}{l}\text { Cisplatin } \\
\text { group }(\mathrm{N}=52)\end{array}$ & $\begin{array}{l}\text { Carboplatin } \\
\text { group }(\mathrm{N}=93)\end{array}$ & $P$-value \\
\hline Median age (range), years & $43(26-65)$ & $47(27-68)$ & 0.478 \\
\hline Menopausal status & & & 0.222 \\
\hline Premenopausal & 35 (67\%) & $53(57 \%)$ & \\
\hline Postmenopausal & 17 (33\%) & $40(43 \%)$ & \\
\hline Clinical tumor stage & & & 0.192 \\
\hline cTI & I (2\%) & $9(10 \%)$ & \\
\hline cT2 & $32(62 \%)$ & $52(55 \%)$ & \\
\hline cT3 & $17(33 \%)$ & $24(26 \%)$ & \\
\hline cT4 & $2(3 \%)$ & $8(9 \%)$ & \\
\hline Clinical lymph node stage & & & 0.231 \\
\hline $\mathrm{cNO}$ & $3(6 \%)$ & $13(14 \%)$ & \\
\hline $\mathrm{cNI}$ & 39 (75\%) & 55 (59\%) & \\
\hline $\mathrm{cN} 2$ & $6(11 \%)$ & $13(14 \%)$ & \\
\hline $\mathrm{cN} 3$ & $4(8 \%)$ & $12(13 \%)$ & \\
\hline Clinical TNM stage & & & 0.831 \\
\hline II & $27(52 \%)$ & $50(54 \%)$ & \\
\hline III & $25(48 \%)$ & $43(46 \%)$ & \\
\hline Ki67 index & & & 0.192 \\
\hline$\geq 20 \%$ & II (2I\%) & $12(13 \%)$ & \\
\hline$<20 \%$ & $4 I(79 \%)$ & 81 (87\%) & \\
\hline Body mass index & & & 0.295 \\
\hline$\geq 25 \mathrm{~kg} / \mathrm{m}^{2}$ & $13(25 \%)$ & 31 (33\%) & \\
\hline$<25 \mathrm{~kg} / \mathrm{m}^{2}$ & $39(75 \%)$ & $62(67 \%)$ & \\
\hline
\end{tabular}

(BMI). The median age was 43 years (range 26-65 years) in the cisplatin group and 47 years (range 27-68 years) in the carboplatin group. A total of 58 patients $(46.9 \%)$ were initially diagnosed with stage III disease, and 88 patients (60.7\%) were premenopausal.

\section{Treatment compliance and surgery}

One hundred and thirty (90\%) patients completed all four cycles of weekly paclitaxel and carboplatin/cisplatin treatment. Because of adverse events, disease progression, withdrawal of consent or immediate surgery, five patients in the cisplatin group and ten patients in the carboplatin group did not finish four cycles. Among these 145 cases, 27 patients received breast conserving surgery, 118 patients had mastectomy, and only two patients received immediate breast reconstruction with the latissimus dorsi muscle flap. Sentinel lymph biopsy was not a common choice for locally advanced breast cancer. Only eight patients received this operation, while nearly $95 \%$ of patients received ALN dissection.

\section{Treatment response}

After two cycles, 132 patients (91\%) had a clinical response (CR or PR). After four cycles, $86 \%$ patients had a clinical response. Two patients in the cisplatin group and five in the carboplatin group received anthracycline, capecitabine or radiotherapy before surgery. The MP scores for responses to four cycles of platinum are listed in Table 2 . The pCR in the carboplatin group was 53\% (ypT0/isNxM0) and $42 \%$ (ypT0/isN0M0) while the pCR in the cisplatin group was $52 \%$ (ypT0/isNxM0) and 44\% (ypT0/isN0M0). In addition, 80 patients who were clinically positive for ALNs had a negative result after NCT. Table 3 lists the distribution of various clinical and pathological characteristics according to the response outcomes. In univariate and multivariate analysis, tumor size, age, ALN status, Ki67 index, and neoadjuvant regimen were not significantly related to $\mathrm{pCR}$. The median follow-up time was 33 months (6-61 months). Survival analyses revealed no significant relationship between regimens and DFS ( $P=0.877$, Figure 1A). Similarly, different kinds of platinum were not significantly related to OS $(P=0.663$, Figure 1B). The Kaplan-Meier curves also demonstrated that age, BMI, tumor size, and lymph node involvement were not prognostic factors; non-pCR was the only significant poor prognostic factor for DFS $(P=0.016)$.

\section{Toxicity}

During the four cycles of NCT, only one patient in the cisplatin group and two patients in the carboplatin group could not

Table 2 Clinical and pathological evaluation

\begin{tabular}{|c|c|c|c|c|}
\hline Variables & $\begin{array}{l}\text { Cisplatin } \\
\text { group }\end{array}$ & $\begin{array}{l}\text { Carboplatin } \\
\text { group }\end{array}$ & OR (95\% Cl) & $P$-value \\
\hline \multicolumn{3}{|l|}{ yрT0/is, ypNo } & $0.911(0.459-1.806)$ & 0.789 \\
\hline No & $29(56 \%)$ & $54(58 \%)$ & & \\
\hline Yes & $23(44 \%)$ & $39(42 \%)$ & & \\
\hline \multicolumn{3}{|c|}{ yрT0/is, ypNO/+ } & I.03I (0.523-2.034) & 0.930 \\
\hline No & $25(48 \%)$ & $44(47 \%)$ & & \\
\hline Yes & $27(52 \%)$ & 49 (53\%) & & \\
\hline \multicolumn{3}{|c|}{ MP grade for breast } & NA & 0.448 \\
\hline MP 5 & $27(52 \%)$ & 49 (53\%) & & \\
\hline MP 4 & $8(15 \%)$ & $8(9 \%)$ & & \\
\hline MP 3 & $14(27 \%)$ & $24(26 \%)$ & & \\
\hline MP 2 & $3(6 \%)$ & $9(10 \%)$ & & \\
\hline MP I & $0(0 \%)$ & $3(3 \%)$ & & \\
\hline \multicolumn{3}{|c|}{ Clinical response after two cycles } & NA & 0.352 \\
\hline $\mathrm{CR}$ & $3(6 \%)$ & $2(2 \%)$ & & \\
\hline PR & $43(82 \%)$ & $84(90 \%)$ & & \\
\hline \multicolumn{5}{|c|}{$(\mathrm{CR}$ or $\mathrm{PR})$} \\
\hline $\mathrm{SD}$ or $\mathrm{PD}$ & $6(12 \%)$ & $7(8 \%)$ & & \\
\hline \multicolumn{3}{|c|}{ Clinical response after four cycles } & NA & 0.570 \\
\hline $\mathrm{CR}$ & $8(15 \%)$ & II (I2\%) & & \\
\hline PR & $37(72 \%)$ & 65 (70\%) & & \\
\hline $\begin{array}{l}\text { Overall } \\
\text { (CR or PR) }\end{array}$ & 45 (87\%) & $76(82 \%)$ & & \\
\hline SD or PD & $2(4 \%)$ & 7 (8\%) & & \\
\hline
\end{tabular}

Notes: ypT0/is, ypN0: the absence of invasive carcinoma in both the breast and axilla. ypT0/is, ypN0/+: the absence of invasive carcinoma in the breast. Abbreviations: $\mathrm{Cl}$, confidence interval; $\mathrm{CR}$, complete response; MP, Miller-Payne; $\mathrm{NA}$, not available; OR, odds ratio; PD, progressive disease; PR, partial response; $\mathrm{SD}$, stable disease. 
Table 3 Odds ratios for pathological complete response according to subgroups

\begin{tabular}{|c|c|c|c|c|c|}
\hline \multirow[t]{2}{*}{ Variables } & \multirow[t]{2}{*}{ Category } & \multicolumn{2}{|l|}{ Univariable } & \multicolumn{2}{|l|}{ Multivariable } \\
\hline & & Odds ratio $(95 \% \mathrm{Cl})$ & $P$-value & Odds ratio $(95 \% \mathrm{Cl})$ & $P$-value \\
\hline Arm & Cisplatin vs carboplatin & $0.911(0.459-1.806)$ & 0.789 & $0.905(0.44 I-I .857)$ & 0.786 \\
\hline Age & $<45$ y vs $\geq 45$ y & $0.724(0.37|-| .4 \mid 4)$ & 0.344 & $0.754(0.376-1.51 \mathrm{I})$ & 0.425 \\
\hline cT & cTI-2 vs cT3-4 & $0.620(0.307-1.252)$ & 0.182 & $0.572(0.278-1.176)$ & 0.129 \\
\hline $\mathrm{cN}$ & cN0-I vs cN2-3 & $0.530(0.237-1.187)$ & 0.123 & $0.487(0.213-1.112)$ & 0.088 \\
\hline Ki67 index & $<20 \%$ vs $\geq 20 \%$ & $2.404(0.887-6.5 \mathrm{I} 2)$ & 0.084 & $2.622(0.945-7.274)$ & 0.064 \\
\hline
\end{tabular}

Abbreviations: $\mathrm{Cl}$, confidence interval; cN, clinical node stage; $\mathrm{cT}$, clinical tumor stage; $y$, years.

tolerate the adverse events and quit the treatment after two cycles. Severe toxicity was also uncommon. The most common grade $3 / 4$ adverse effect was hematological toxicity, such as neutropenia and leukopenia (Table 4). Grade 3/4 non-hematological toxicity was rare, and only diarrhea and peripheral neuropathy occurred. There was no significant difference between the adverse events in the groups.

\section{Discussion}

Because of the younger age, poorly differentiated tumors, and shortened survival, TNBC is a high-risk breast cancer that lacks the benefit of targeted therapies. In metastatic breast cancer patients, previous clinical trials have indicated that the addition of platinum salts to chemotherapy can increase the response rate or progression free survival (PFS). ${ }^{8,10}$

The BRCA1/2 mutation is associated with DNA damage, and platinum can cause DNA damage. In a retrospective analysis of 6,903 patients, including 102 patients with the germ-line BRCA1 mutation, the highest pCR rate was reported in germ-line BRCA1-mutation carriers who received neoadjuvant cisplatin therapy (83\%). In the other treatment subgroups, such as anthracyclines and taxanes or cyclophosphamide, methotrexate and fluorouracil, a low rate of pCR was observed in women with breast cancer and BRCA1 mutation. ${ }^{11}$ However, combining the two trials, the pCR in patients with BRCA mutation and wild-type TNBC was similar. ${ }^{12}$ BRCA mutated cancers and some sporadic TNBC have a potentially higher sensitivity to DNA-damaging agents, such as platinum salts.

In untreated metastatic breast cancer, single platinum agents have had encouraging overall response rates ( $42 \%-54 \%$ for cisplatin, $32 \%$ for carboplatin). ${ }^{13}$ In prospective clinical trials, the addition of carboplatin increased the pCR rates from $28 \%$ to $42 \%$ in stage II-III TNBC. ${ }^{14}$ The addition of weekly carboplatin to non-pegylated liposomal doxorubicin, weekly paclitaxel and bevacizumab boosted the pCR rate from $38 \%$ to $58 \% .{ }^{15}$ In a meta-analysis including 28 studies, the pooled rate of pCR for cisplatin was $41.9 \%$ (95\% CI: $32 \%-51 \%$ ) while carboplatin was associated with a pCR rate of $46.3 \%(95 \%$ CI: $40.7 \%-52.1 \%) .{ }^{16}$ In a retrospective analysis for platinum salt in TNBC, the study found that cisplatin offered a survival advantage over carboplatin in both PFS and OS. Cisplatin/docetaxel neoadjuvant therapy was well tolerated and an effective therapy in locally advanced TNBC ${ }^{17}$ Patients who achieved pCR had a significant benefit in relapse and death compared with non-pCR patients. However, we lack long-term outcome data from prospective

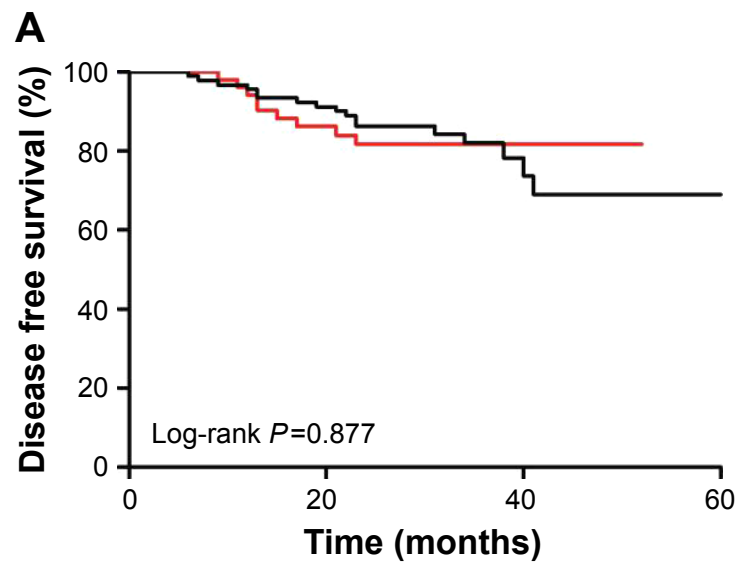

B

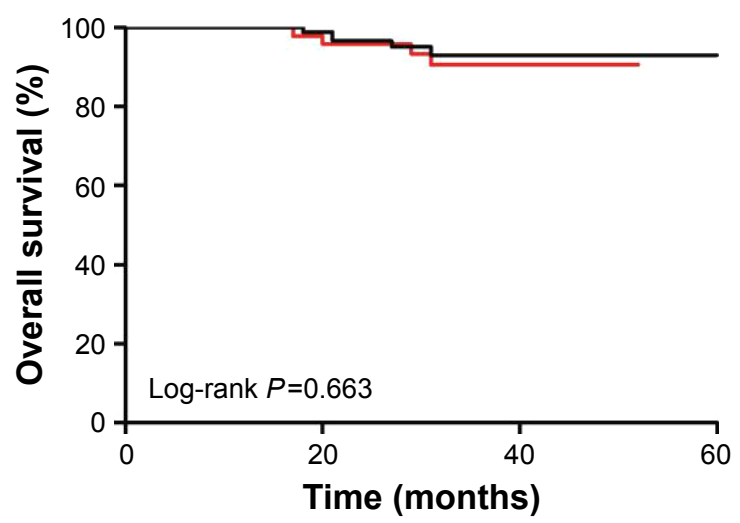

- Cisplatin group - Carboplatin group

Figure I Kaplan-Meier disease free survival curve $(\mathbf{A})$ and overall survival curve $(\mathbf{B})$ of triple negative breast cancer patients treated with cisplatin or carboplatin. 
Table 4 Most common adverse events reported as possibly, probably, or definitely related to treatment

\begin{tabular}{|c|c|c|c|c|}
\hline \multirow[t]{2}{*}{ Adverse event } & \multicolumn{2}{|c|}{$\begin{array}{l}\text { Cisplatin group } \\
(\mathrm{N}=52)\end{array}$} & \multicolumn{2}{|c|}{$\begin{array}{l}\text { Carboplatin group } \\
(\mathrm{N}=93)\end{array}$} \\
\hline & Grade I/2 & Grade 3/4 & Grade $1 / 2$ & Grade 3/4 \\
\hline Anemia & $26(50 \%)$ & $5(10 \%)$ & $55(59 \%)$ & $8(9 \%)$ \\
\hline Febrile neutropenia & $0(0 \%)$ & $3(6 \%)$ & $0(0 \%)$ & $5(5 \%)$ \\
\hline Neutropenia & $21(40 \%)$ & $31(60 \%)$ & $25(27 \%)$ & $68(73 \%)$ \\
\hline Leukopenia & 22 (42\%) & $26(50 \%)$ & 30 (32\%) & 55 (59\%) \\
\hline Thrombocytopenia & 19 (37\%) & $3(6 \%)$ & 31 (33\%) & 7 (8\%) \\
\hline Bilirubin increased & $23(44 \%)$ & $3(6 \%)$ & 37 (40\%) & $3(3 \%)$ \\
\hline AST increased & 17 (33\%) & $2(4 \%)$ & $25(27 \%)$ & $2(2 \%)$ \\
\hline ALT increased & 13 (25\%) & I (2\%) & $28(30 \%)$ & $0(0 \%)$ \\
\hline Diarrhea & $8(15 \%)$ & I (2\%) & $13(14 \%)$ & I (I\%) \\
\hline Nausea/vomiting & 23 (44\%) & I (2\%) & 38 (4I\%) & $0(0 \%)$ \\
\hline Arthralgia/myalgia & $3(6 \%)$ & $0(0 \%)$ & $9(10 \%)$ & $0(0 \%)$ \\
\hline Fatigue & $26(50 \%)$ & $0(0 \%)$ & 39 (42\%) & $\mathrm{I}(\mathrm{I} \%)$ \\
\hline Peripheral neuropathy & 7 (13\%) & $2(4 \%)$ & $5(5 \%)$ & $0(0 \%)$ \\
\hline
\end{tabular}

Abbreviations: ASL, aspartate transaminase; ALT, alanine transaminase.

neoadjuvant trials to determine if the addition of different types of platinum salts and the higher pCR rates associated with its addition significantly impacted DFS or OS. These results indicate that adding effective agents, such as antiangiogenics, poly-ADP ribose polymerase (PARP) inhibitors, and other small molecule inhibitors, which may increase the pCR rate, is a potential cure for TNBC patients. ${ }^{18-20}$

Cisplatin is highly protein bound and leads to high drug levels in the kidneys, liver, and other organs, slowing renal excretion and contributing to its side effect profile, most of which are dose related. On the other hand, carboplatin remains largely unbound to plasma proteins and the serum concentration is closely linked to renal clearance. In toxicity analysis, cisplatin typically causes more nausea and vomiting, nephrotoxicity, and neurotoxicity, while carboplatin is more likely to cause myelosuppression. ${ }^{21}$ However, severe anemia and thrombocytopenia only occurred in less than $5 \%$ of patients after four cycles of carboplatin in previous studies. ${ }^{6,22}$ After four cycles of single-agent cisplatin, grade $3 / 4$ hematological and non-hematological adverse events (AE) occurred in less than $5 \%$ of patients. ${ }^{23}$ When combination cisplatin and paclitaxel was used, grade III neutropenia, anemia, nausea, and vomiting were reported in $8 \%-9 \%$ of Western patients. ${ }^{24}$ In our study, the grade $3 / 4 \mathrm{AE}$ were neutropenia and leukopenia and were over $50 \%$, while AE of anemia and thrombocytopenia were less than $10 \%$ in both groups. Nausea/vomiting and peripheral neuropathy was slightly higher in the cisplatin group but did not reach significance.

Both types of platinum salts and weekly paclitaxel are feasible therapies that achieved high $\mathrm{pCR}$ rates, similar survival rate, and tolerability in TNBC patients. We recognize that the research still has some points of weakness. First, this was a retrospective study that lacked long-term adverse event observations and long-term invalid survival improvement. A further limitation of our study is the small number of patients from which to draw meaningful conclusions. The best platinum salt to be added to NAC is still unknown. Further studies are needed on the comparison of different platinum combinations and the comparison between carboplatin- and cisplatin-based combinations. Based on biomarkers identified by high throughput technology with DNA, RNA or proteins, researchers will be able to find a subgroup of patients who will receive the greatest benefit.

\section{Acknowledgment}

This research was supported by the National Natural Science Foundation of China ( 81502289 and 81302298 ). The funders had no role in the study design, data collection and analysis, decision to publish, or preparation of the manuscript.

\section{Author contributions}

All authors contributed toward data analysis, drafting and critically revising the paper, gave final approval of the version to be published, and agree to be accountable for all aspects of the work.

\section{Disclosure}

The authors report no conflicts of interest in this work.

\section{References}

1. Abramson VG, Lehmann BD, Ballinger TJ, Pietenpol JA. Subtyping of triple-negative breast cancer: implications for therapy. Cancer. 2015;121(1):8-16.

2. Lehmann BD, Bauer JA, Chen X, Sanders ME, Chakravarthy AB, Shyr Y, Pietenpol JA. Identification of human triple-negative breast cancer subtypes and preclinical models for selection of targeted therapies. J Clin Invest. 2011;121(7):2750-2767.

3. Cortazar P, Zhang L, Untch M, et al. Pathological complete response and long-term clinical benefit in breast cancer: the CTNeoBC pooled analysis. Lancet. 2014;384(9938):164-172.

4. Liedtke C, Mazouni C, Hess KR, et al. Response to neoadjuvant therapy and long-term survival in patients with triple-negative breast cancer. J Clin Oncol. 2008;26(8):1275-1281.

5. Carey LA, Dees EC, Sawyer L, et al. The triple negative paradox: primary tumor chemosensitivity of breast cancer subtypes. Clin Cancer Res. 2007;13(8):2329-2334

6. Chen XS, Nie XQ, Chen CM, et al. Weekly paclitaxel plus carboplatin is an effective nonanthracycline-containing regimen as neoadjuvant chemotherapy for breast cancer. Ann Oncol. 2010;21(5):961-967.

7. Cortazar P, Zhang L, Untch M, et al. Abstract P1-14-20: Meta-analysis Results from the Collaborative Trials in Neoadjuvant Breast Cancer (CTNeoBC). Cancer Research. 2012;72(24 Suppl):S1-S11.

8. Petrelli F, Barni S, Bregni G, de Braud F, Di Cosimo S. Platinum salts in advanced breast cancer: a systematic review and meta-analysis of randomized clinical trials. Breast Cancer Res Treat. 2016;160(3): $425-437$. 
9. Ogston KN, Miller ID, Payne S, et al. A new histological grading system to assess response of breast cancers to primary chemotherapy: prognostic significance and survival. Breast. 2003;12(5):320-327.

10. $\mathrm{Hu} \mathrm{XC,} \mathrm{Zhang} \mathrm{J,} \mathrm{Xu} \mathrm{BH,} \mathrm{et} \mathrm{al.} \mathrm{Cisplatin} \mathrm{plus} \mathrm{gemcitabine} \mathrm{versus} \mathrm{pacli-}$ taxel plus gemcitabine as first-line therapy for metastatic triple-negative breast cancer (CBCSG006): a randomised, open-label, multicentre, phase 3 trial. Lancet Oncol. 2015;16(4):436-446.

11. Byrski T, Gronwald J, Huzarski T, et al. Pathologic complete response rates in young women with BRCA1-positive breast cancers after neoadjuvant chemotherapy. J Clin Oncol. 2010;28(3):375-379.

12. Sharma P, Lopez-Tarruella S, Garcia-Saenz JA, et al. Efficacy of neoadjuvant carboplatin plus docetaxel in triple negative breast cancer: Combined analysis of two cohorts. Clin Cancer Res. 2017;23(3): 649-657.

13. Decatris MP, Sundar S, O'Byrne KJ. Platinum-based chemotherapy in metastatic breast cancer: current status. Cancer Treat Rev. 2004; 30(1):53-81.

14. Sikov WM, Berry DA, Perou CM, et al. Impact of the addition of carboplatin and/or bevacizumab to neoadjuvant once-per-week paclitaxel followed by dose-dense doxorubicin and cyclophosphamide on pathologic complete response rates in stage II to III triple-negative breast cancer: CALGB 40603 (Alliance). J Clin Oncol. 2015;33(1):13-21.

15. von Minckwitz G, Schneeweiss A, Loibl S, et al. Neoadjuvant carboplatin in patients with triple-negative and HER2-positive early breast cancer (GeparSixto; GBG 66): a randomised phase 2 trial. Lancet Oncol. 2014;15(7):747-756.

16. Petrelli F, Coinu A, Borgonovo K, Cabiddu M, Ghilardi M, Lonati V, Barni S. The value of platinum agents as neoadjuvant chemotherapy in triple-negative breast cancers: a systematic review and meta-analysis. Breast Cancer Res Treat. 2014;144(2):223-232.
17. Hurley J, Reis IM, Rodgers SE, et al. The use of neoadjuvant platinumbased chemotherapy in locally advanced breast cancer that is triple negative: retrospective analysis of 144 patients. Breast Cancer Res Treat. 2013;138(3):783-794.

18. Llombart-Cussac A, Bermejo B, Villanueva C, et al. SOLTI NeoPARP: a phase II randomized study of two schedules of iniparib plus paclitaxel versus paclitaxel alone as neoadjuvant therapy in patients with triple-negative breast cancer. Breast Cancer Res Treat. 2015;154(2): 351-357.

19. von Minckwitz G, Muller BM, Loibl S, et al. Cytoplasmic poly(adenosine diphosphate-ribose) polymerase expression is predictive and prognostic in patients with breast cancer treated with neoadjuvant chemotherapy. J Clin Oncol. 2011;29(16):2150-2157.

20. Ma X, Wang X, Huang J, et al. Bevacizumab addition in neoadjuvant treatment increases the pathological complete response rates in patients with HER-2 negative breast cancer especially triple negative breast cancer: a meta-analysis. PLoS One. 2016;11(8):e0160148.

21. Sikov WM. Assessing the role of platinum agents in aggressive breast cancers. Curr Oncol Rep. 2015;17(2):3.

22. Sikov WM, Dizon DS, Strenger R, et al. Frequent pathologic complete responses in aggressive stages II to III breast cancers with every-4-week carboplatin and weekly paclitaxel with or without trastuzumab: a Brown University Oncology Group Study. J Clin Oncol. 2009;27(28):4693-4700.

23. Silver DP, Richardson AL, Eklund AC, et al. Efficacy of neoadjuvant Cisplatin in triple-negative breast cancer. J Clin Oncol. 2010;28(7): 1145-1153.

24. Ezzat AA, Ibrahim EM, Ajarim DS, et al. Phase II study of neoadjuvant paclitaxel and cisplatin for operable and locally advanced breast cancer: analysis of 126 patients. Br J Cancer. 2004;90(5):968-974.
OncoTargets and Therapy

\section{Publish your work in this journal}

OncoTargets and Therapy is an international, peer-reviewed, open access journal focusing on the pathological basis of all cancers, potential targets for therapy and treatment protocols employed to improve the management of cancer patients. The journal also focuses on the impact of management programs and new therapeutic agents and protocols on

\section{Dovepress}

patient perspectives such as quality of life, adherence and satisfaction The manuscript management system is completely online and includes a very quick and fair peer-review system, which is all easy to use. Visit http://www.dovepress.com/testimonials.php to read real quotes from published authors. 